\title{
CLAVES DE IDENTIFICACIÓN PARA LAS ESPECIES IBÉRICAS DEL GÉNERO COELIOXYS LATREILLE, 1809 (HYMENOPTERA, APOIDEA, MEGACHILIDAE) ${ }^{1}$
}

\author{
F. J. Ortiz-Sánchez*, F. Torres** \& C. Ornosa***
}

\begin{abstract}
RESUMEN
El trabajo actual, dentro de la revisión que está en curso sobre la familia Megachilidae en la fauna ibérica, incluye las claves de identificación para las 20 especies del género Coelioxys Latreille, 1809 que, según el conocimiento actual, habitan en nuestra Península. Las claves se acompañan de dibujos ilustrativos que permiten su reconocimiento.

Palabras clave: Hymenoptera, Megachilidae, Coelioxys, claves de identificación, especies ibéricas.
\end{abstract}

\begin{abstract}
Keys to the Iberian species of the genus Coelioxys Latreille, 1809 (Hymenoptera, Apoidea, Megachilidae)

This paper includes keys to the 20 Iberian species of the genus Coelioxys Latreille, 1809 (Megachilidae), as part of the ongoing general revision of the taxa of that family of bees that inhabit our Peninsula. The accompanying illustrations should make identifi-
\end{abstract} cation possible.

Key words: Hymenoptera, Megachilidae, Coelioxys, keys, Iberian species.

\section{Introducción}

Coelioxys Latreille, 1809 es un género de abejas cleptoparásitas de distribución casi cosmopolita que contiene un alto número de especies, entre 320 y 350. Probablemente encuentra su máxima diversidad en Suramérica (Michener, 2007). Si bien la clasificación subgenérica presenta distintas perspectivas según que el género sea estudiado desde la óptica de los especialistas del Viejo o el Nuevo Mundo, hay que destacar el esfuerzo de, por ejemplo, Schwarz (1999) o Přidal \& Tkalců (2001) por aclarar algunas situaciones controvertidas. En el territorio mediterráneo occidental existen 4 subgé-

\footnotetext{
Subvencionado por el Proyecto CGL2007-60877

* Grupo de Investigación "Transferencia de I+D en el Área de Recursos Naturales". Universidad de Almería. Ctra. de Sacramento, s/n. E-04120 La Cañada de San Urbano (Almería), España. E-mail: fjortiz@ual.es

** Departamento de Biología Animal, Ecología, Parasitología y Edafología. Facultad de Biología. Universidad de Salamanca. Campus Miguel de Unamuno, s/n. E-37071 Salamanca, España. E-mail: torres@usal.es

*** Departamento de Zoología y Antropología Física. Facultad de Biología. Universidad Complutense. c/ José Antonio Nováis, 2. E-28040 Madrid, España. E-mail: paddy@bio.ucm.es
} 
neros representados por 22 especies. En la Península Ibérica se encuentran 3 de esos subgéneros (Allocoelioxys Tkalců, 1974, que cuenta con 10 especies en este territorio, Coelioxys, con 9 especies, y Mesocoelioxys Ruszkowski, 1986, con sólo 1) (Ornosa et al., 2007; Schwarz, 2009). El cuarto (Liothyrapis Cockerell, 1911) está presente en toda África (excepto en el Sahara), además de en el área mediterránea oriental, desde donde su distribución se extiende hacia el este por la región transcaspiana y el sur de Asia (Michener, 2007).

La primera (y hasta el momento única) clave para la identificación de las especies españolas data de hace más de un siglo (Dusmet, 1906) y forma parte del conjunto de artículos que ese autor publicó sobre la fauna española de Apoidea. Aquel trabajo, que supuso una aportación valiosa, dada la inexistencia de obras específicas para nuestra fauna, adolecía de descripciones ambiguas y, sobre todo, del desconocimiento de parte de las especies de nuestra geografía, así como de sus sinonimias. Esto llevó al autor a cometer inevitables errores que, a veces, inducen a una identificación incorrecta. Existen obras recientes sobre los Megachilidae de diversas partes de Europa, que incluyen claves para las especies de este género (Scheuchl, 1996; Banaszak \& Romasenko, 2001; Amiet et al., 2004), pero ninguna es aplicable a la Península Ibérica, por ser la nuestra una fauna mucho más rica en lo que respecta a este grupo. El trabajo actual, consecuencia de la revisión que se está llevando a cabo sobre la fauna ibérica de Megachilidae, comprende las claves de identificación de los géneros y especies ibéricos. Las claves se acompañan de dibujos ilustrativos.

\section{Material y métodos}

Para realizar este estudio se han revisado las colecciones depositadas en las siguientes instituciones: Museo Nacional de Ciencias Naturales de Madrid, Departamento de Biología Animal, Ecología, Parasitología y Edafología de la Universidad de Salamanca, Departamento de Zoología y Antropología Física de la Universidad Complutense de Madrid y la colección particular de uno de los autores (F. J. Ortiz-Sánchez), así como diversos ejemplares de comparación cedidos en préstamo por Maximilian Schwarz (Ansfelden, Austria).

DESCRIPCIÓN DEL GÉNERO Y CARACTERES DIAGNÓSTICOS

Como es general para el conjunto de los Apoidea, los ejemplares de Coelioxys, sobre el tegumento, están provistos de pubescencia o pilosidad, constituida por setas de una longitud variable según la zona del cuerpo. Normalmente tienen forma de pelo, delgado, largo y más o menos erecto, en la cabeza y el tórax; son más gruesas, cortas y tumbadas, en el tórax y el gáster; o presentan aspecto escuamiforme, "escamas", que aparecen sobre todo en el gáster, donde pueden dar lugar a manchas triangulares en las esquinas de los escleritos, formar bandas completas o incluso cubrir amplias áreas del tegumento.

Las hembras tienen las antenas compuestas por escapo, pedicelo y 10 flagelómeros; el escapo es cilíndrico y más grueso que el flagelo. Los flagelómeros son aproximadamente tan largos como anchos y, en algunas especies, pueden ser desde parduscos a rojizos en su cara ventral. En este trabajo, al igual que en otros, se adopta la convención de nombrar los elementos antenales por la abreviatura A seguida del ordinal en subíndice, de modo que el escapo es denominado como $\mathrm{A}_{1}$, y los sucesivos como $\mathrm{A}_{2}, \mathrm{~A}_{3}$, etc. La cabeza es más ancha que larga, con contorno elíptico o algo rectangular. Todas las especies de nuestra fauna presentan los ojos compuestos cubiertos en toda su superficie por quetas cortas y erectas. Las mandíbulas presentan 3 dientes. En el tórax, el escutelo tiene en cada axila una espina dirigida hacia atrás, con diferente longitud y forma según las especies; el postescutelo es inerme. El primer par de alas tiene estigma y dos celdas submarginales; el ápice de la marginal está alejado del borde anterior del ala. Las patas son de tipo marchador típico sin modificaciones apreciables, a excepción de que en algunas especies existe una escotadura ancha en la base de los fémures de las patas intermedias; también puede presentarse una espina o diente, con desarrollo variable, sobre las coxas del primer par de patas; las tibias carecen de placa basitibial y los tarsos de arolio. El gáster presenta 6 segmentos visibles externamente; no existe área pigidial ni rima; la forma general es cónica, con su mayor anchura en el segundo segmento. Como es usual, se designa a terguitos y esternitos, abreviadamente, por su inicial, es decir, $\mathrm{T}$ y $\mathrm{E}$, respectivamente, seguida de un subíndice que corresponde al ordinal del segmento gastral referido, con lo que, por ejemplo, el tercer terguito se abrevia como $\mathrm{T}_{3}$ y el quinto esternito como $\mathrm{E}_{5} . \mathrm{T}_{1}$ es ampliamente cóncavo; su base en declive es más lisa y brillante que el disco y la unión entre ambos es aguda. $\mathrm{T}_{6}$ y $\mathrm{E}_{6}$ normalmente están prolongados, más el esternito (a veces encorvado hacia abajo) que el terguito, y con escultura y forma de gran importancia taxonómica. Por lo general, el tegu- 


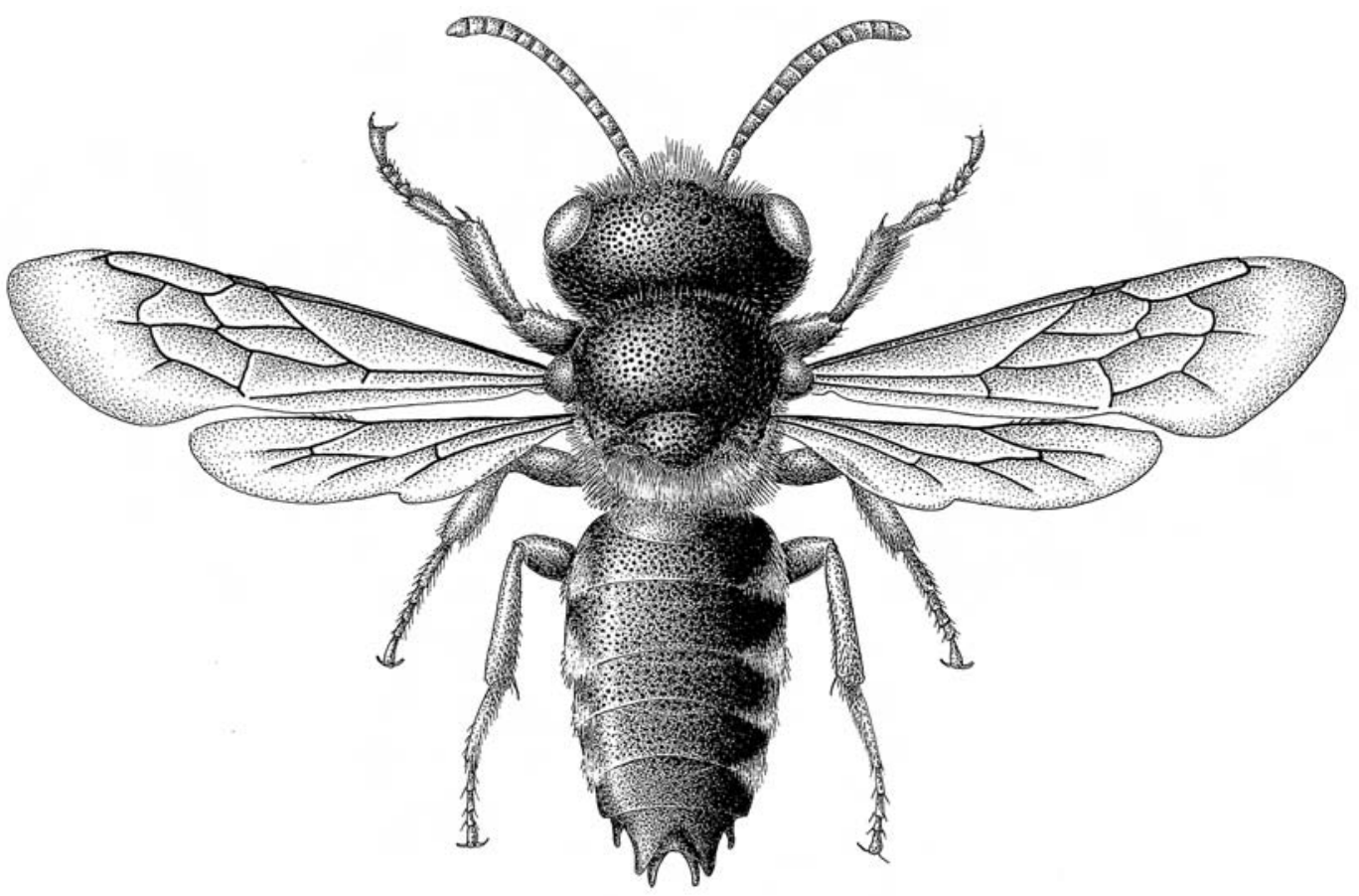

Fig. 1.- Vista general del macho de Coelioxys inermis.

Fig. 1.- General view of the male of Coelioxys inermis.

mento es de color negro completamente, pero algunas especies presentan zonas coloreadas de rojo, sobre todo en el primer y último segmento del gáster y en distinta extensión de las patas, mandíbulas $\mathrm{y}$ antenas. El punteado general es grueso, profundo y espeso; en zonas como las genas y el mesonoto puede formar arrugas y, generalmente, está más dispersamente dispuesto y es más superficial en el gáster. Los esternitos siempre son lisos, siendo su escultura más delicada y dispersa que la de los terguitos. El pelo es de una longitud variable según la zona del cuerpo; normalmente es delgado, largo y más o menos erecto, en la cabeza y el tórax; también aparece pelo más grueso, corto y tumbado, normalmente en tórax y gáster; finalmente, hay pelo escamoso que aparece sobre todo en el gáster, formando manchas triangulares en las esquinas de los escleritos, bandas completas, o incluso cubriendo amplias áreas del cuerpo. No existe escopa en los esternitos. La longitud oscila entre 6,5 y $15 \mathrm{~mm}$.

Los machos (Fig. 1) tienen las antenas formadas por escapo, pedicelo y 11 artejos flagelares; el escapo es cilíndrico, más grueso que el flagelo. La cabeza es cuadrada o, normalmente, más ancha que larga. Los ojos compuestos están densamente cubiertos por quetas cortas. Las mandíbulas presen$\tan 3$ dientes. En la zona inferior de cada gena, junto al espacio malar, existe un área lampiña, más o menos lisa y brillante, a veces rebordeada, conocida como "superficie lampiña subgenal". El tórax (y todo el gáster, a excepción de su extremo distal) está conformado como en las hembras, así como las alas. Las patas son marchadoras, sin modificaciones, a excepción de una espina que, con mayor desarrollo que en las hembras, aparece, según subgéneros, sobre cada coxa del primer par de patas; las tibias no presentan placa basitibial ni los tarsos arolio. En el gáster hay 7 terguitos y 6 esternitos visibles ( $\mathrm{T}_{7}$ y $\mathrm{E}_{6}$ a menudo cubiertos por el respectivo esclerito precedente), sin área pigidial ni rima. En algunas especies, $T_{2}$ presenta, en cada giba, una fóvea a modo de foseta rodeada de punteado y con forma más o menos oval, con importancia taxonómica. $\mathrm{T}_{6}$ está provisto de un variado número de espinas, existiendo un par distal, otro par en el ángulo proximal $\mathrm{y}$, al menos, otro par en posición dorsal, que puede estar fusionado o subdividido; la superficie que queda entre las espinas más distales suele ser más o menos cóncava, y aquí se la abrevia como "concavidad". Por lo general, el tegumento 
es completamente negro, a excepción de algunos casos en los que las patas y algunos segmentos gastrales están teñidos de color rojo. Los esternitos son como en las hembras, si bien $\mathrm{E}_{4}$ puede estar escotado en el centro del borde apical y $\mathrm{E}_{6}$ nunca está prolongado. También el pelo es en general como en las hembras. La longitud oscila entre 6 y $14 \mathrm{~mm}$.

\section{Resultados}

\section{Lista de las especies ibéricas del género Coelioxys Latreille, 1809}

\author{
Subgénero Allocoelioxys Tkalců, 1974 \\ C. (A.) acanthura (Illiger, 1806) \\ C. (A.) afra Lepeletier, 1841 \\ C. (A.) brevis Eversmann, 1852 \\ C. (A.) caudata Spinola, 1838 \\ C. (A.) coturnix Pérez, 1883 \\ C. (A.) echinata Förster, 1853 \\ C. (A.) emarginata Förster, 1853 \\ C. (A.) haemorrhoa Förster, 1853 \\ C. (A.) obtusa Pérez, 1883 \\ C. (A.) polycentris Förster, 1853
}

Subgénero Coelioxys Latreille, 1809

C. (C.) aurolimbata algeriensis Friese, 1895

C. (C.) conoidea (Illiger, 1806)

C. (C.) elongata Lepeletier, 1841

C. (C.) inermis (Kirby, 1802)

C. (C.) lanceolata Nylander, 1852

C. (C.) mandibularis Nylander, 1848

C. (C.) osmiae Alfken, 1928

C. (C.) quadridentata (Linnaeus, 1758)

C. (C.) rufescens Lepeletier \& Serville, 1825

Subgénero Mesocoelioxys Ruszkowski, 1986

C. (M.) argentea Lepeletier, 1841

\section{Clave de subgéneros}

\section{Machos}

1. Cuerpo provisto de pelos, sin escamas (especialmente visible en las bandas distales de los terguitos, que están formadas por pelos gruesos cortos y tumbados, pero no propiamente escamosos). Coxas del primer par de patas con una espina larga y roma, normalmente aplastada. $\mathrm{T}_{6}$ con 3 pares de espinas (Figs. 7-11), 2 centrales sobre una protuberancia y el otro en los ángulos

Coelioxys

- Cuerpo provisto de pelos y escamas (éstas son especialmente visibles en las bandas distales de los terguitos). Coxas del primer par de patas sin espinas o, más raramen- te, con ellas. $\mathrm{T}_{6}$ con 4 pares de espinas (Figs. 2-6) o, más raramente, 3 (Fig. 12B)

2. Coxas del primer par de patas provistas de una espina; $T_{6}$ con 3 pares de espinas (Fig. 12B). Longitud: 11-14 mm .. Mesocoelioxys

- Coxas del primer par de patas desprovistas de espinas; $\mathrm{T}_{6}$ con 4 pares de espinas (Figs. 2-6). Normalmente especies menores Allocoelioxys

\section{Hembras}

1. Cuerpo provisto de pelos, sin escamas (especialmente visible en las bandas distales de los terguitos, que están formadas por pelos gruesos cortos y tumbados, pero no propiamente escamosos). Tubérculos pronotales (= callos humerales) sin borde en quilla lameliforme. Coxas del primer par de patas con un proceso en espina corta y roma, plana Coelioxys

- Cuerpo provisto de pelos y escamas (éstas son especialmente visibles en las bandas distales de los terguitos). Tubérculos pronotales con borde en quilla lameliforme. Coxas del primer par de patas sin espinas o, más raramente, con ellas 2

2. Coxas del primer par de patas provistas de una espina. Longitud: $12,5-15 \mathrm{~mm}$ Mesocoelioxys

- Coxas del primer par de patas desprovistas de espinas. Normalmente especies menores Allocoelioxys

\section{Subgénero Allocoelioxys}

\section{Machos}

1. $\mathrm{T}_{7}$ largo y agudo, extendido por debajo de $\mathrm{T}_{6}$. Éste posee una pubescencia apretada de escamas que forma faja en la parte basal y queda interrumpida por una quilla central longitudinal brillante, sobre una giba, que discurre desde la base hasta la concavidad (Fig. 2C). Longitud: 11-13 mm

C. (A.) acanthura

- $\mathrm{T}_{7}$ normal o, a lo sumo, acabado en ángulo, pero no sobresaliente por debajo de $\mathrm{T}_{6}$. Éste con pubescencia dispersa o con manchas o banda de escamas densas, sin quilla de esas características

2. $\mathrm{T}_{7}$ agudo pero no sobresaliente bajo $\mathrm{T}_{6}$. Éste está densamente cubierto de escamas tumbadas en el centro y en toda la concavidad; los 2 pares de espinas superiores son cortos y se hallan prácticamente al mismo nivel (Fig. 4B). Suelen presentar color rojo las mandíbulas, antenas, patas y último segmento gastral. Cara ventral del cuerpo ampliamente cubierta de escamas. Especie pequeña: 6,5-8 mm

C. (A.) coturnix

- Sin esas características reunidas. Especies normalmente mayores ................................................................ 3

3. $\mathrm{T}_{6}$ alargado, con las 6 espinas centrales cortas, romas y próximas entre sí y alejadas del par exterior; las del par central inferior no están plenamente separadas, dando al terguito un aspecto distal bilobulado en vez de bífido (Figs. $5 \mathrm{~F}$ y $6 \mathrm{~B}$ )

- $\mathrm{T}_{6}$ de longitud normal; espinas agudas y separadas ........ 5 
A
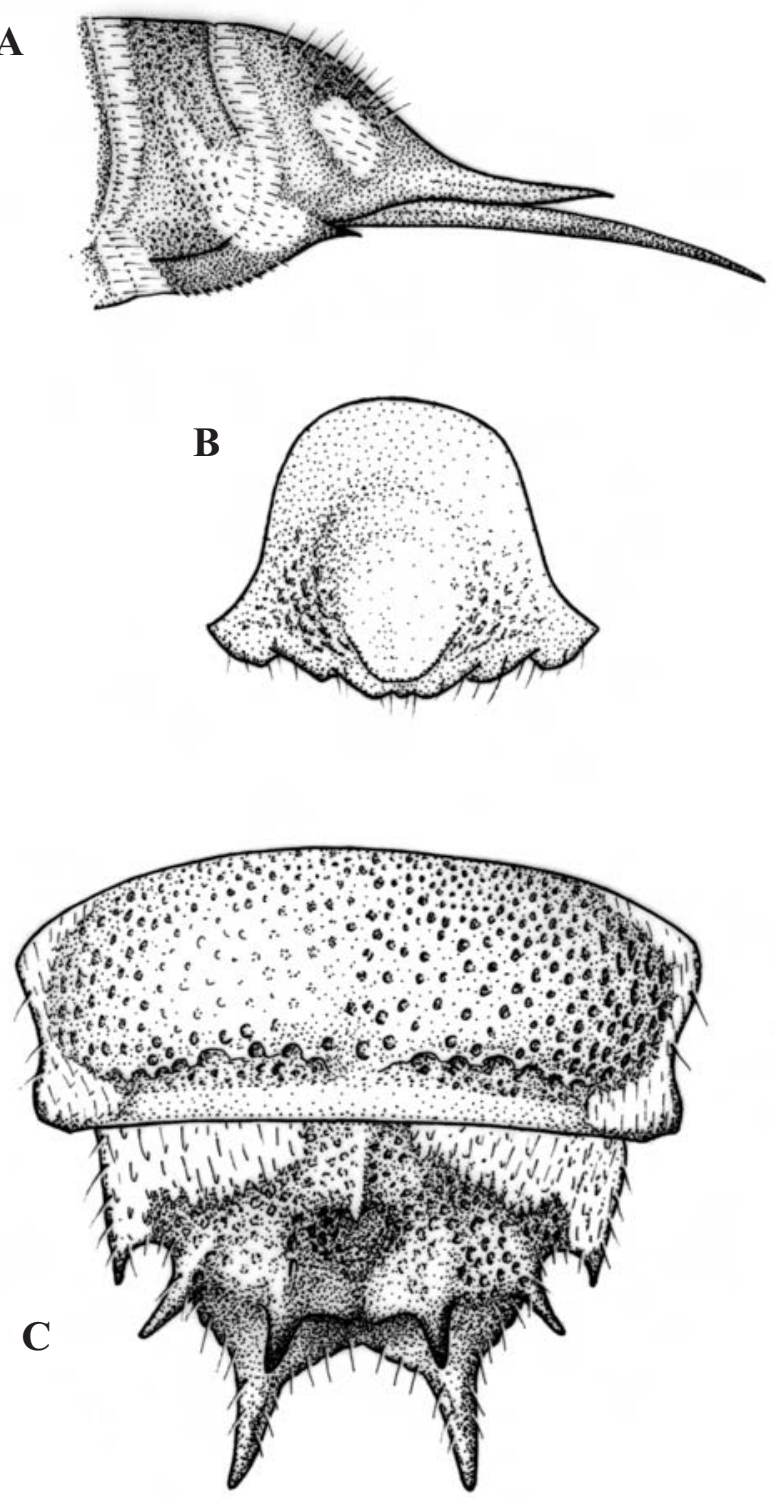
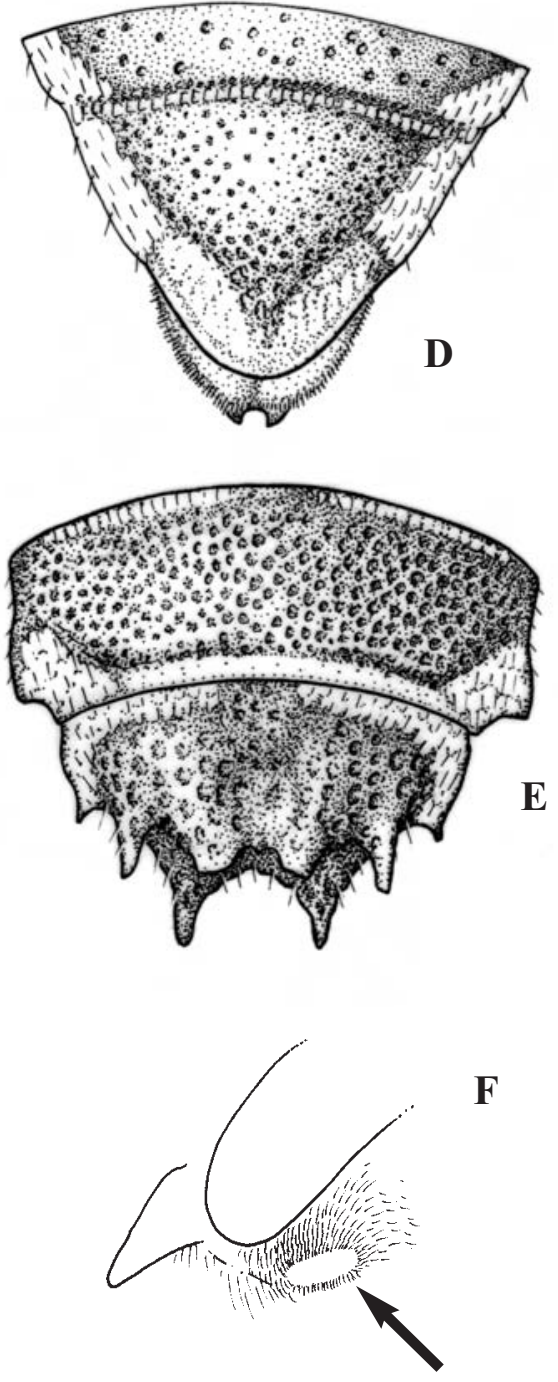

Fig. 2.- Coelioxys (A.) acanthura: extremo del gáster (A) y clípeo (B) de la 우 y extremo del gáster del $\bigcirc^{\top}$ (C); C. (A.) afra: extremo del gáster de la + (D) y del $\sigma^{7}(\mathrm{E})$ y superficie subgenal del $O^{7}(\mathrm{~F})$.

Fig. 2.-Coelioxys (A.) acanthura: apex of the gaster (A) and clypeus (B) of the $ᄋ$ and apex of the gaster of the $\sigma^{\pi}(\mathrm{C})$; $C$. (A.) afra: apex of the gaster of the $O$ (D) and of the $\sigma^{7}(\mathrm{E})$ and subgenal surface of the $\sigma^{7}(\mathrm{~F})$.

4. Las 2 espinas centrales superiores son cortas y triangulares, y están situadas por encima de las dos latero-superiores (Fig. 5F). Mandíbulas, antenas y patas negras. Longitud: 8,5-10 mm C. (A.) polycentris

- Las 2 espinas centrales superiores (que son triangulares y cortas) están situadas más abajo que las dos laterales superiores (que son más largas y agudas; Fig. 6B). Mandíbulas, antenas y patas rojas. Longitud: 7,5-9 $\mathrm{mm}$

C. (A.) obtusa
5. Antenas, mandíbulas y espinas inferiores de $\mathrm{T}_{6}$ rojas. La superficie lampiña subgenal es elíptica, oblicua al margen del ojo, y su extremo inferior no forma reborde sobresaliente; el superior con punteado grueso. $\mathrm{T}_{6}$ con una mancha semicircular de escamas apretadas, sin giba en la base. $\mathrm{T}_{5}$ con pequeños dientes laterales romos (Fig. 5D). Especie pequeña: $6,5-8 \mathrm{~mm}$

C. (A.) haemorrhoa

- Sin esas características reunidas. Especies normalmente mayores 

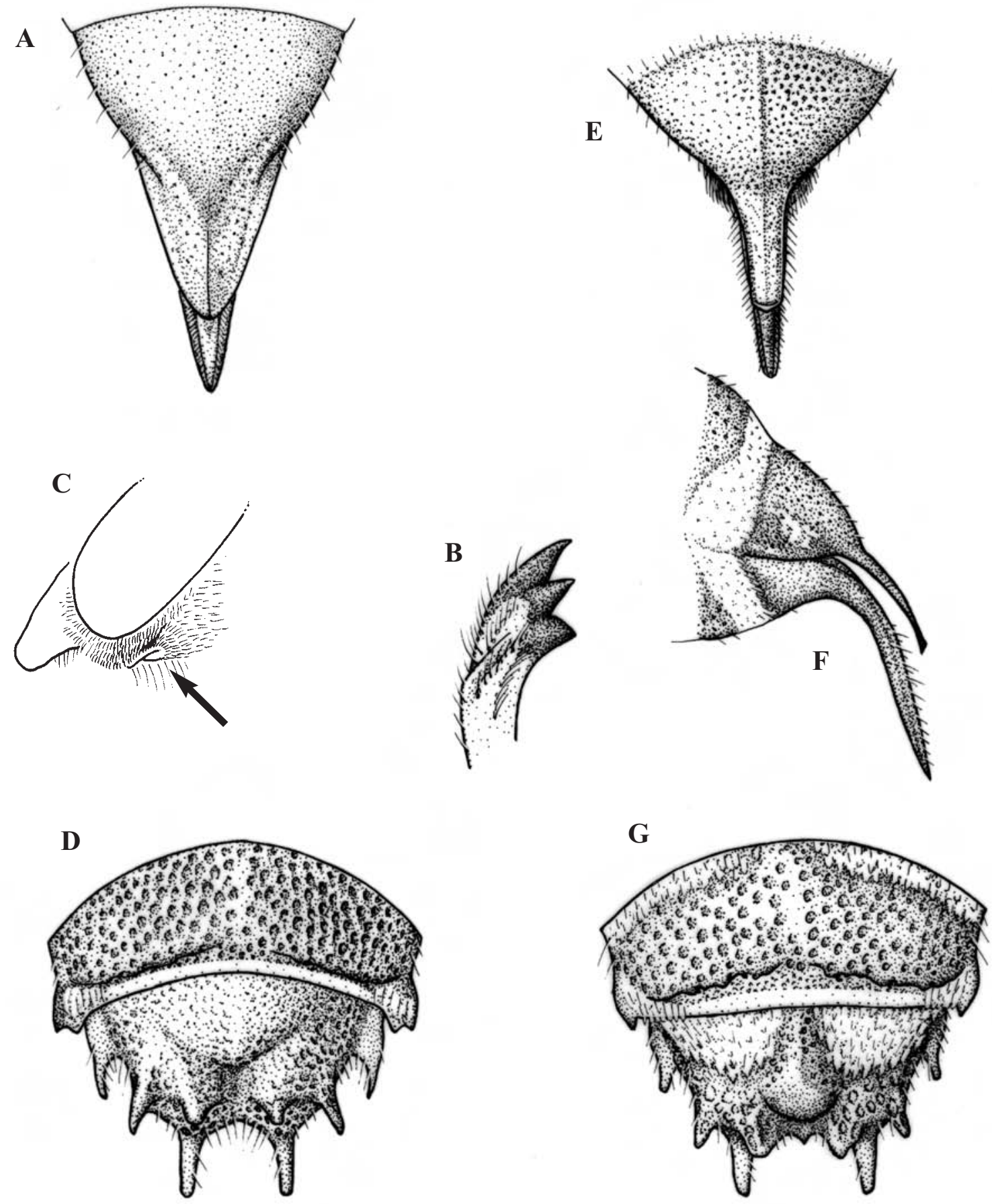

Fig. 3.-Coelioxys (A.) brevis: extremo del gáster (A) y mandíbula (B) de la $\$$ y superficie subgenal (C) y extremo del gáster (D) del $\sigma^{x} ;$ C. (A.) caudata: extremo del gáster de la $ᄋ$ (E, F) y del $\sigma^{\pi}(\mathrm{G})$.

Fig. 3.-Coelioxys (A.) brevis: apex of the gaster (A) and mandible (B) of the + and subgenal surface (C) and apex of the gaster (D) of the $\sigma^{x} ;$ C. (A.) caudata: apex of the gaster of the $O$ (E, F) and of the $\sigma^{x}(\mathrm{G})$. 

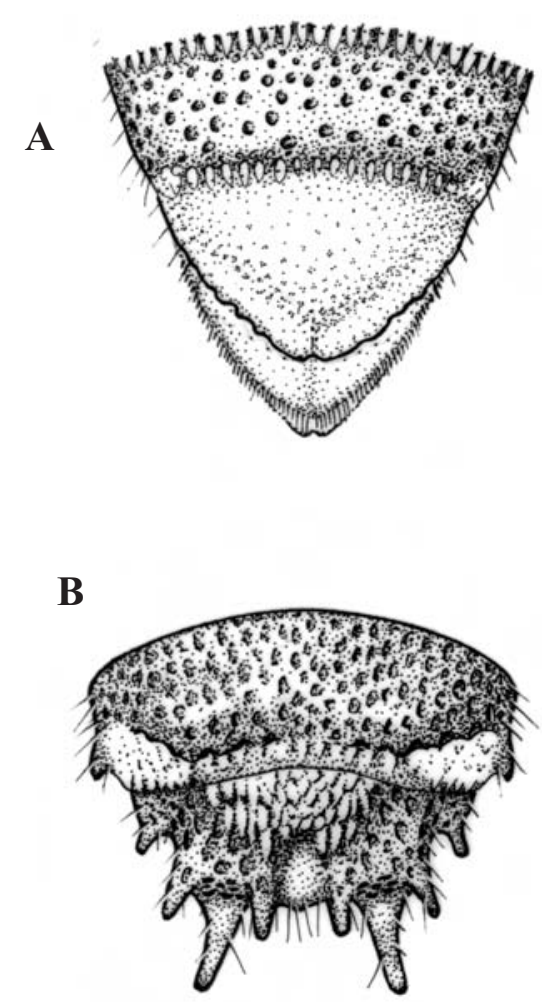
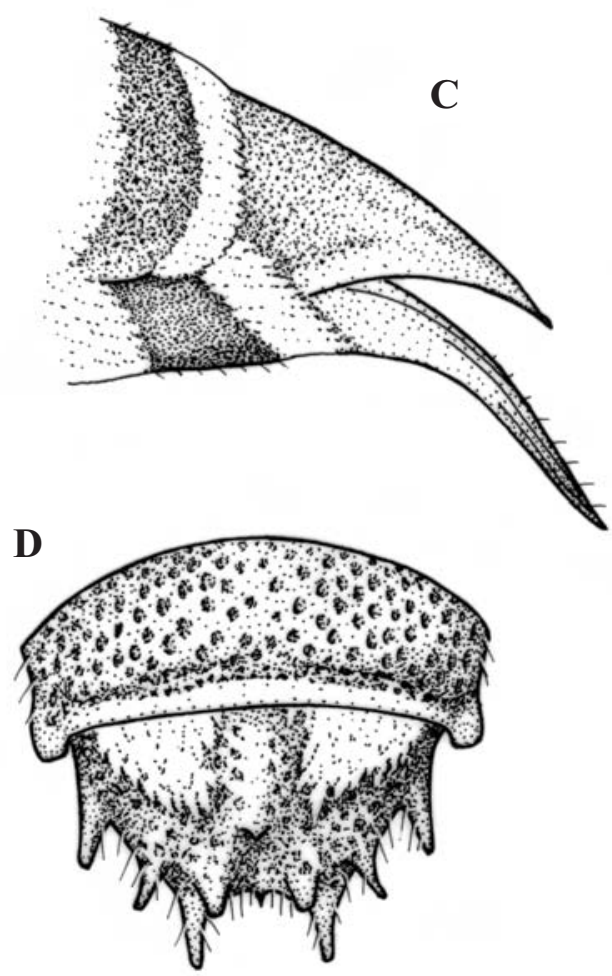

Fig. 4.-Coelioxys (A.) coturnix: extremo del gáster de la 우 (A) y del O (B); C. (A.) echinata: ídem (C, D).

Fig. 4.-Coelioxys (A.) coturnix: apex of the gaster of the $ᄋ$ (A) and of the $\sigma^{\top}(\mathrm{B}) ;$ C. (A.) echinata: the same (C, D).

6. Especie mayor: 10-12 mm. Antenas negras por la cara ventral. Esternitos con fajas distales anchas y enteras que cubren las depresiones, sin pubescencia en el disco. Borde distal de $\mathrm{E}_{4}$ con escote triangular en el centro. La superficie lampiña subgenal está mal delimitada y en parte oculta por los pelos largos contiguos a la cavidad oral. Extremo del gáster como en la figura 5B

C. (A.) emarginata

- Especies menores. Antenas rojizas o parduscas por la cara ventral. Esternitos con pubescencia en el disco; si hay fajas, suelen estar difuminadas desde el borde distal hacia el disco. Superficie lampiña subgenal bien delimitada y rodeada de pubescencia corta

7. $\mathrm{E}_{4}$ sin escotadura en el centro del borde apical. $\mathrm{T}_{6}$ provisto de una giba en el centro de su base; ampliamente cubierto de escamas, interrumpidas por la giba, que llegan lateralmente a la concavidad. $\mathrm{T}_{5}$, en cada ángulo, con diente ancho y romo (Fig. 4D). Bandas distales de los terguitos estrechas en el centro (pueden estar interrumpidas), formadas por 1-2 filas de escamas. Superficie lampiña subgenal pequeña, plana, no rebordeada por delante, y con puntos en su extremo posterior. Longitud: $6-8,5 \mathrm{~mm}$

C. (A.) echinata

- $\mathrm{E}_{4}$ con una escotadura más o menos profunda en el centro del borde apical. Bandas distales de los terguitos más anchas en el centro, formadas por 2-3 filas de escamas. Especies ligeramente mayores

\section{8}

8. $\mathrm{T}_{6} \sin$ giba, con caída continua desde la base hasta la concavidad; las espinas infero-laterales son muy cortas y las centro-superiores casi no están diferenciadas, son cortas y romas. $\mathrm{T}_{5}$ anguloso en los lados pero sin formar espina (Fig. 2E). $\mathrm{E}_{4}$ con una escotadura triangular en el centro del borde apical, que está deprimido. Superficie lampiña subgenal completamente lisa y brillante, muy ligeramente cóncava y algo sobreelevada por delante, pero sin formar reborde (Fig. 2F). Antenas rojo amarillentas por la cara ventral. Esternitos con anchas fajas distales de escamas interumpidas en el centro. Longitud: 6,5-8,5 mm

C. (A.) afra

- $\mathrm{T}_{6}$ con una giba más o menos desarrollada (Figs. 3D y $3 \mathrm{G}$ ). Superficie lampiña subgenal cóncava y, por delante, sobresaliente en reborde (Fig. 3C)

\section{9}

9. $\mathrm{T}_{6}$ con una giba central longitudinal, larga, que en el centro puede constituir una quilla roma y llega a tapar, en su extremo, la mayor parte de la concavidad; espinas inferolaterales más cortas y triangulares, pero agudas en su extremo. $T_{5}$ con extremos angulosos pero sin formar dientes sobresalientes (Fig. 3G). Escotadura de $\mathrm{E}_{4}$ triangular, deli- 
A

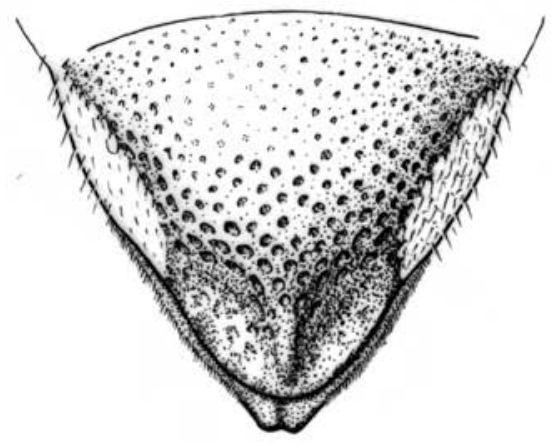

C

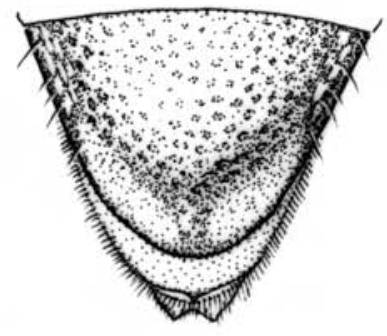

$\mathbf{E}$

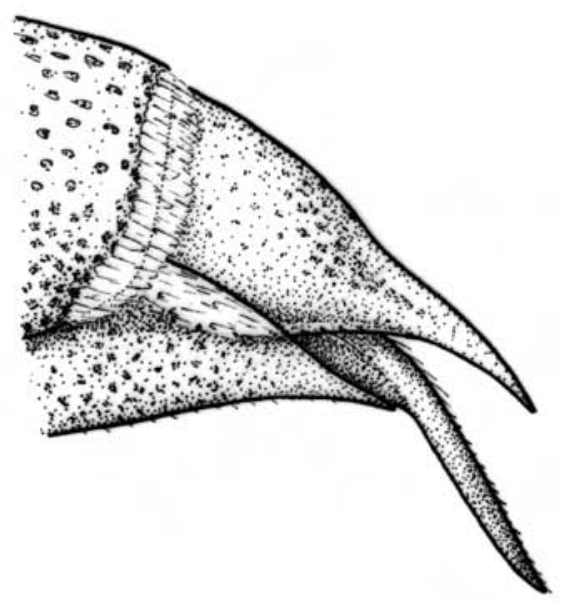

B

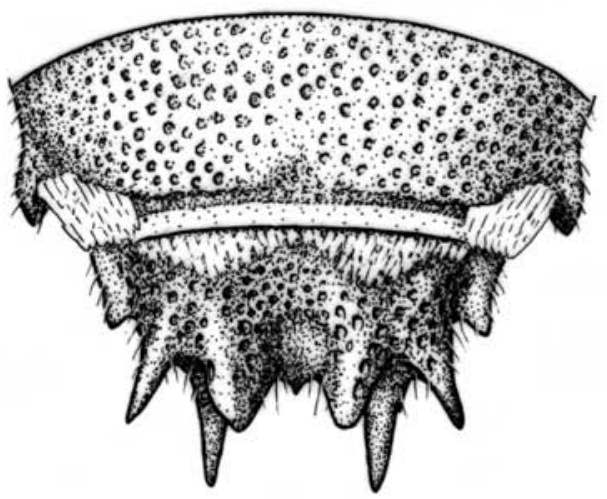

D

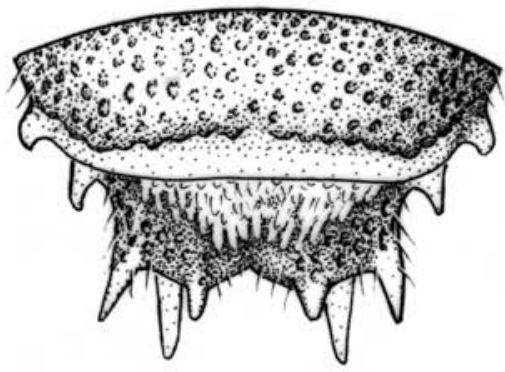

F

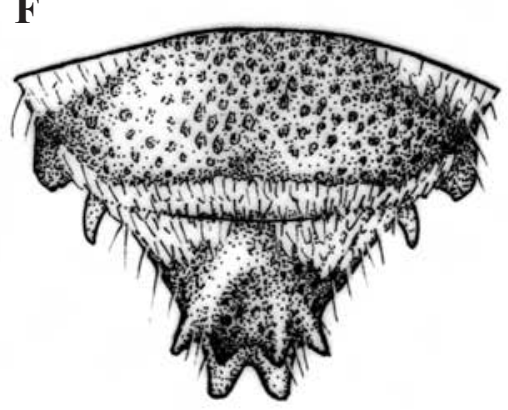

Fig. 5.-Coelioxys (A.) emarginata: extremo del gáster de la $\odot$ (A) y del Oヤ (B); C. (A.) haemorrhoa: ídem (C, D); C. (A.) polycentris: ídem (E, F).

Fig. 5.-Coelioxys (A.) emarginata: apex of the gaster of the 9 (A) and of the $\sigma^{7}$ (B); C. (A.) haemorrhoa: the same (C, D); C. (A.) polycentris: the same (E, F). 
A

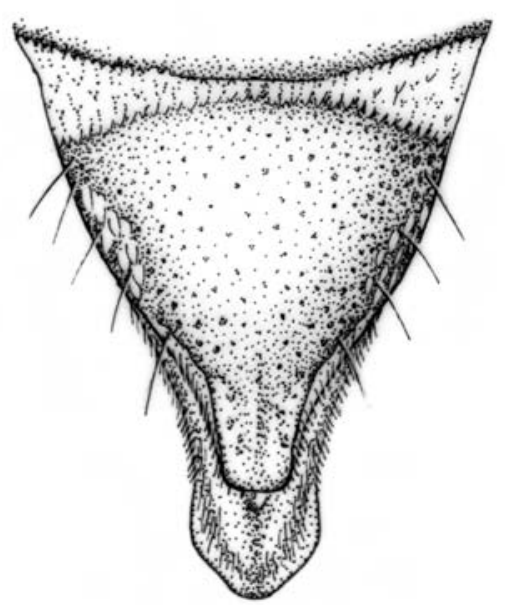

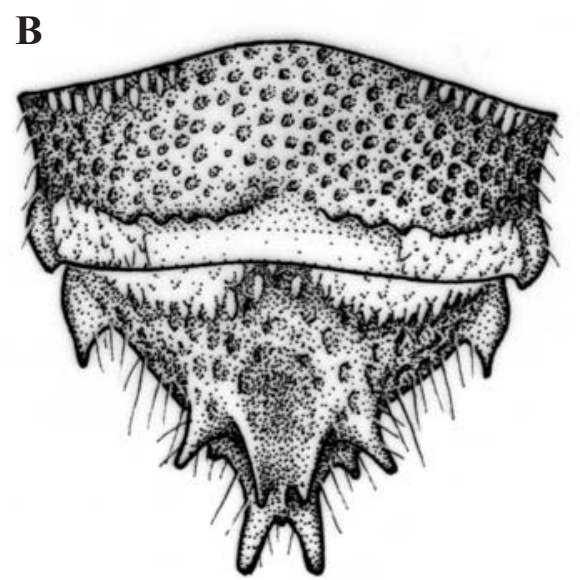

Fig. 6.-Coelioxys (A.) obtusa: extremo del gáster de la 우 (A) y del $\bigcirc^{7}$ (B).

Fig. 6.- Coelioxys (A.) obtusa: apex of the gaster of the 9 (A) and of the $\sigma^{x}$ (B).

mitada por un par de pequeños dientes obtusos. Longitud: 7,5-9,5 mm C. (A.) caudata

- $\mathrm{T}_{6}$ con una giba corta en la base y una concavidad amplia; sus espinas ínfero-laterales son largas y delgadas. $\mathrm{T}_{5}$ con dientes laterales diferenciados, romos, con aspecto bilobulado (Fig. 3D). Escotadura de $\mathrm{E}_{4}$ muy poco marcada pero amplia y delimitada por un par de pequeños dientes agudos. Longitud: 7-9,5 mm

C. (A.) brevis

\section{Hembras}

1. Región distal de $\mathrm{T}_{6} \mathrm{y} \mathrm{E}_{6}$ alargada, estrecha. La prolongación de $\mathrm{E}_{6}$ es larga $\mathrm{y}$, generalmente, encorvada, $1,5-2$ veces más larga que la parte apical de $\mathrm{T}_{6} . \mathrm{E}_{6} \mathrm{y} \mathrm{T}_{6}$ son subiguales en anchura .....

2

- Región distal de $\mathrm{T}_{6}$ y E 6 ancha y corta. La prolongación de $\mathrm{E}_{6}$ es aproximadamente la mitad de larga que la parte apical de $\mathrm{T}_{6} . \mathrm{E}_{6}$ es más ancho que $\mathrm{T}_{6}$

2. $\mathrm{T}_{6} \mathrm{y} \mathrm{E}_{6}$ moderada y progresivamente estrechados hacia el extremo; la prolongación de $\mathrm{E}_{6}$ tiene los lados casi paralelos (Figs. 3A, 4C y 5E)

- $\mathrm{T}_{6}$ y $\mathrm{E}_{6}$ fuertemente estrechados en la mitad de su longitud, con una parte distal estrecha en forma de hoja de espada (Figs. 2A y 3E)

3. Últimos segmentos gastrales negros. $\mathrm{E}_{6}$ bruscamente encorvado hacia abajo y con una escotadura distal redondeada (Fig. 5E). $\mathrm{T}_{1}$ a $\mathrm{T}_{5}$ con bandas distales anchas (2-3 filas de escamas), no tan estrechadas en el centro. Longitud: 9-10 mm ....

C. (A.) polycentris

- $\mathrm{T}_{6}$ con, al menos, el ápice rojo. $\mathrm{E}_{6}$ completamente rojo, encorvado progresivamente y sin escotadura distal. $\mathrm{T}_{2}$ a $\mathrm{T}_{5}$ con bandas apicales anchas a los lados, pero estrechadas en el centro
4. Mandíbulas más robustas y cuadrangulares, algo arqueadas en la base, con fuerte quilla en el segundo diente (Fig. 3B). Gena inferior estrecha abajo, la quilla occipital discurre en diagonal hasta el margen inferior del ojo compuesto. $\mathrm{E}_{6}$ es completamente rojo; $\mathrm{T}_{6}$ rojo sólo en su mitad distal, y presenta manchas laterales afieltradas de color blanco. Bandas distales de los terguitos anchas en el centro, formadas por 2-3 filas de escamas. Antenas por la cara ventral, y patas, de color rojo oscuro. $\mathrm{E}_{2}$ a $\mathrm{E}_{4}$ con fajas apicales anchas interrumpidas en el centro. Extremo del gáster como en la figura 3A. Longitud: $7-10 \mathrm{~mm}$ C. (A.) brevis

- Mandíbulas sin modificaciones. Gena inferior ancha, la quilla occipital casi paralela al margen lateral del ojo compuesto. $\mathrm{E}_{6}$ es rojo; $\mathrm{T}_{6}$ completamente negro o con el extremo rojo, y no presenta manchas blancas afieltradas laterales o éstas son minúsculas. Bandas distales de los terguitos estrechas en el centro, formadas por una fila de escamas. Antenas negras. Patas parduscas. Esternitos con anchas fajas completas. Extremo del gáster como en la figura 4C. Longitud: $8,5-10 \mathrm{~mm}$

C. (A.) echinata

5. $\mathrm{T}_{6}$ y $\mathrm{E}_{6}$ agudos, en forma de hoja de espada (Fig. 2A). Clípeo y mandíbulas pardo rojizos; el primero muy convexo en el centro, formando una especie de giba cuyo extremo alcanza prácticamente el del clípeo (Fig. 2B). Longitud: $13-15 \mathrm{~mm}$

C. (A) acanthura

- $\mathrm{T}_{6}$ ancho en el extremo; $\mathrm{E}_{6}$ redondeado estrechamente o con una ligera escotadura distal. Clípeo y mandíbulas negros; el primero más o menos moderadamente conve-

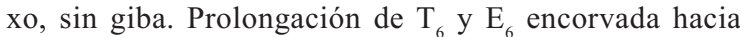
abajo (Figs. 3E y 3F). Longitud: $9-11 \mathrm{~mm}$

\section{C. (A.) caudata}

6. $\mathrm{E}_{6}$ acabado en ángulo romo, no truncado ni escotado .... 7

- $\mathrm{E}_{6}$ abruptamente truncado o ligeramente escotado en el extremo 

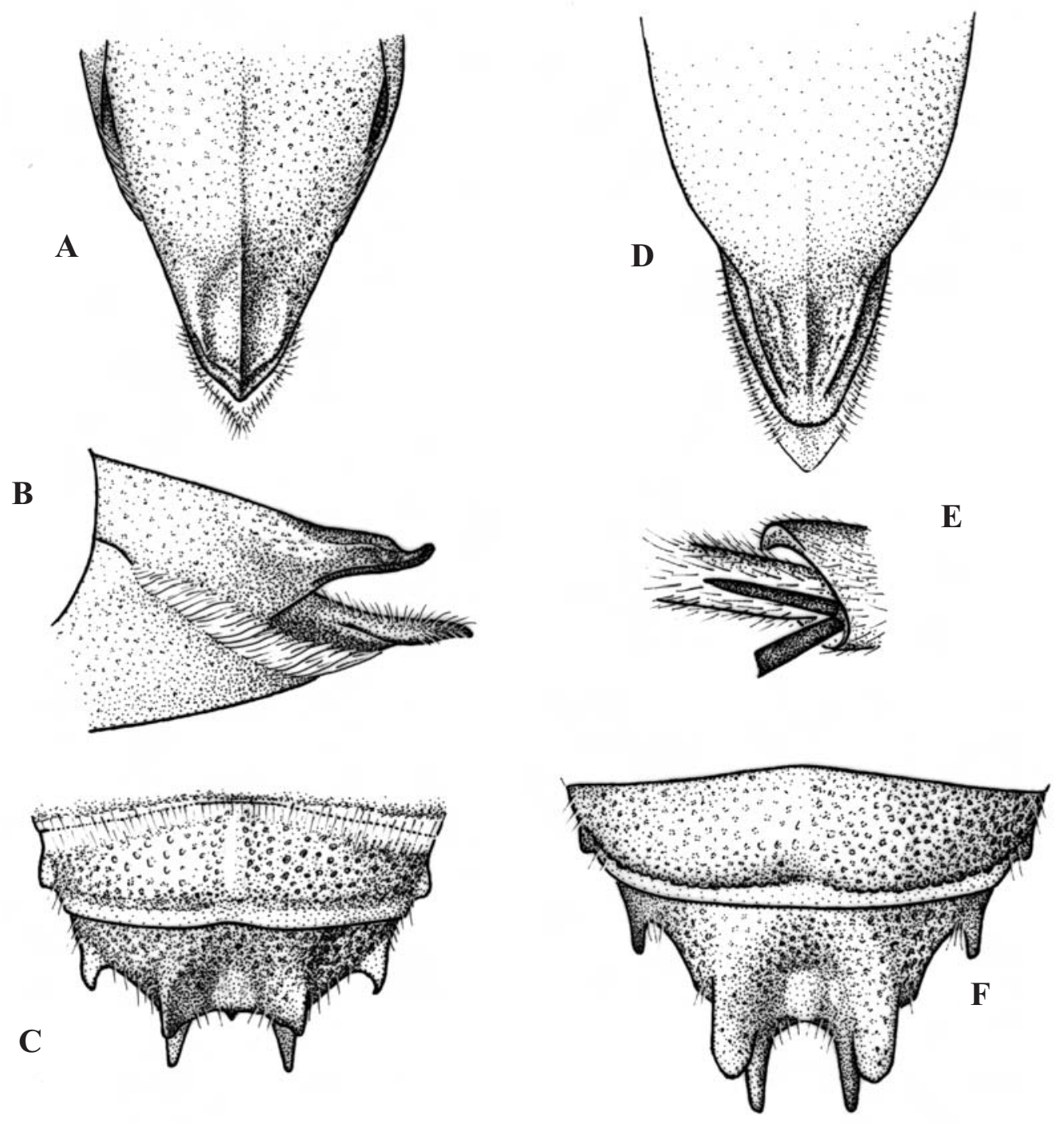

Fig. 7.- Coelioxys (C.) aurolimbata algeriensis: extremo del gáster de la 우 (A, B) y del O (C); C. (C.) conoidea: extremo del gáster (D) y espolones tibiales traseros de la $ᄋ$ (E) y extremo del gáster del $\mathrm{O}^{7}(\mathrm{~F})$.

Fig. 7.- Coelioxys (C.) aurolimbata algeriensis: apex of the gaster of the 우 (A, B) and of the $\sigma^{7}(\mathrm{C})$; C. (C.) conoidea: apex of the gaster (D) and tibial spurs of the hind leg of the $\$(\mathrm{E})$ and apex of the gaster of the $\sigma^{x}(\mathrm{~F})$.

7. $\mathrm{E}_{6}$ con los lados progresivamente convergentes, con el extremo triangular en un ángulo agudo (Fig. 4A). Área supraclipear y frente, entre las inserciones antenales, provistas de una ligera quilla central longitudinal. Longitud: 7$8 \mathrm{~mm}$ C. (A.) coturnix

- $\mathrm{E}_{6}$ con los lados paralelos preapicalmente, con el extremo triangular en ángulo recto (Fig. 6A). Área supraclipear y frente, entre las inserciones antenales, desprovistas de quilla central longitudinal. Longitud: $8,5-10 \mathrm{~mm}$

\section{C. (A.) obtusa}

8. Especie grande: $11-13 \mathrm{~mm}$. Todos los segmentos gastrales negros o, a lo sumo, con tono pardusco. Antenas y mandíbulas negras o parduscas. Terguitos gastrales con amplias manchas escamosas triangulares y una banda estrecha completa formada por 1 fila de escamas. Extremo del gáster como en la figura $5 \mathrm{~A} \ldots \ldots \ldots \ldots \ldots \ldots . . . . . . . .(A$.$) emarginata$ - Especies menores: 8-9 mm. Antenas y mandíbulas, en mayor o menor parte de su superficie, rojizas, así como $\mathrm{T}_{6}$ $\mathrm{y} \mathrm{E}_{6}$ 

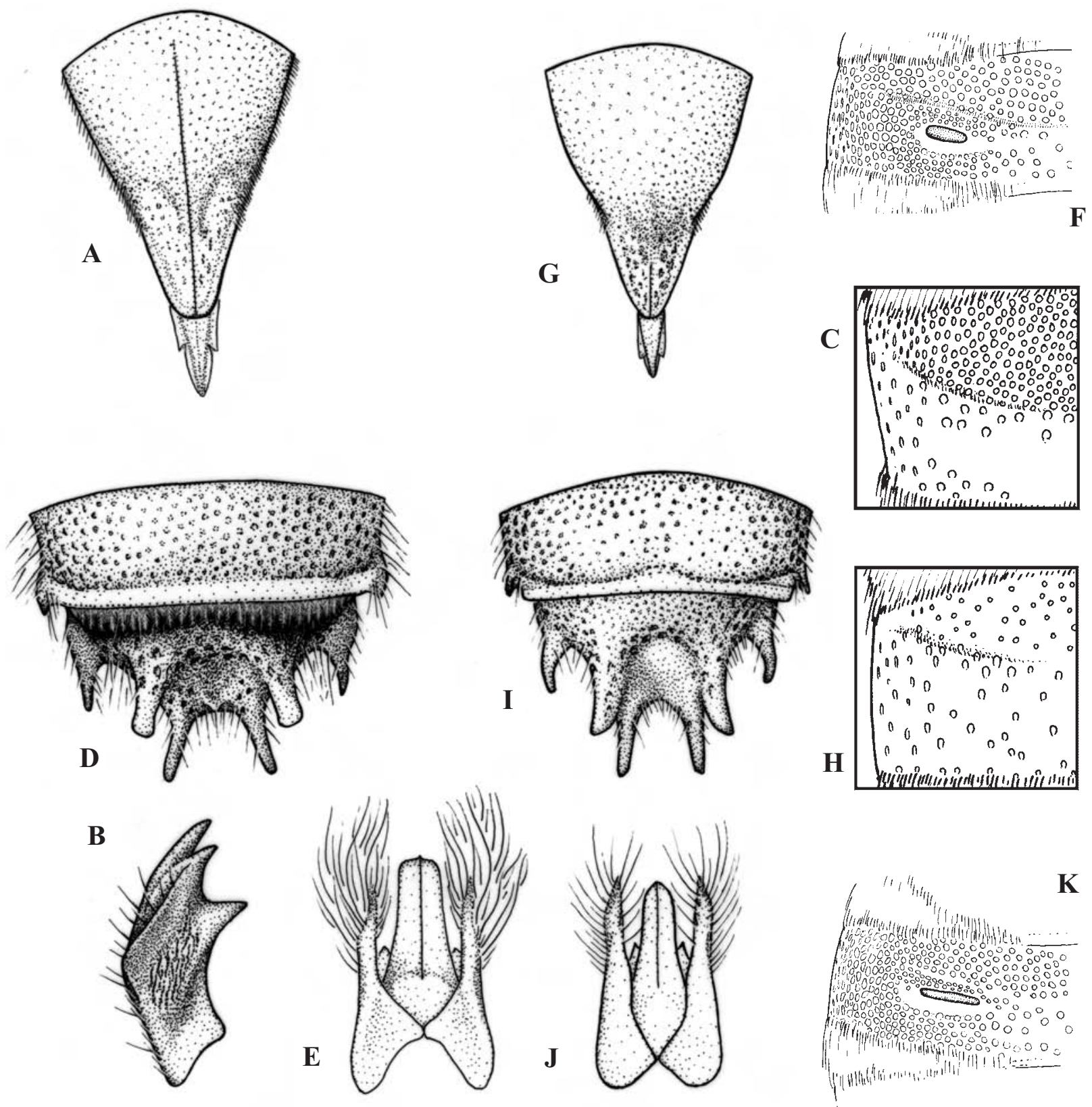

Fig. 8.-Coelioxys (C.) elongata: extremo del gáster (A), mandíbula (B) y $\mathrm{T}_{2}(\mathrm{C})$ de la $\$$ y extremo del gáster (D), genitalia (E) y $_{2}(\mathrm{~F})$ del $\mathrm{O}^{\mathrm{T}}$; C. (C.) inermis: extremo del gáster $(\mathrm{G})$ y $\mathrm{T}_{2}$ de la $ᄋ(\mathrm{H})$ y extremo del gáster $(\mathrm{I})$, genitalia $(\mathrm{J})$ y $\mathrm{T}_{2}(\mathrm{~K})$ del $\mathrm{O}^{\mathrm{T}}$.

Fig. 8.- Coelioxys (C.) elongata: apex of the gaster (A), mandible (B) and $\mathrm{T}_{2}(\mathrm{C})$ of the $ᄋ$ and apex of the gaster (D), genitalia (E) and $\mathrm{T}_{2}(\mathrm{~F})$ of the $\mathrm{O}^{\prime} ;$ C. (C.) inermis: apex of the gaster $(\mathrm{G})$ and $\mathrm{T}_{2}$ of the $\mathrm{O}(\mathrm{H})$ and apex of the gaster (I), genitalia (J) and $\mathrm{T}_{2}(\mathrm{~K})$ of the $\mathrm{O}^{\mathrm{T}}$. 


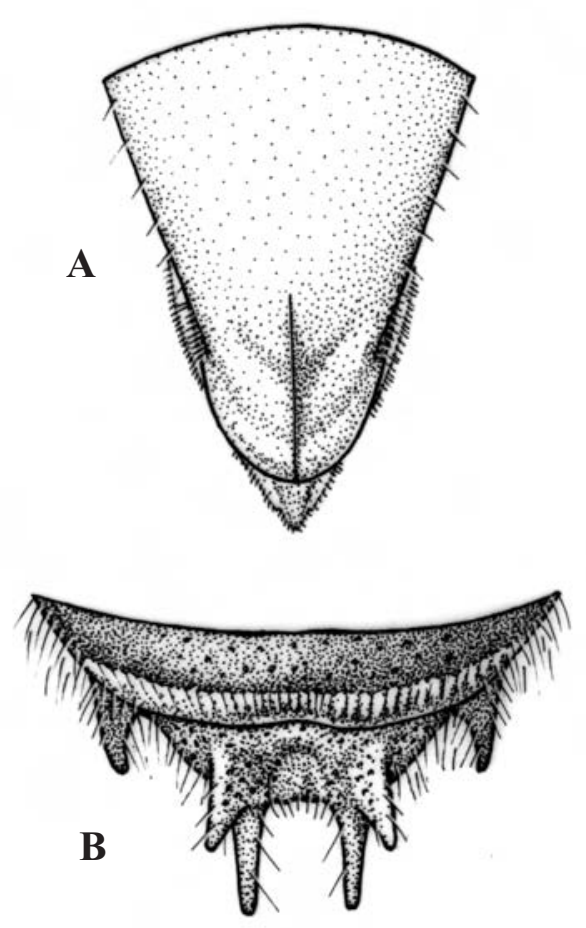

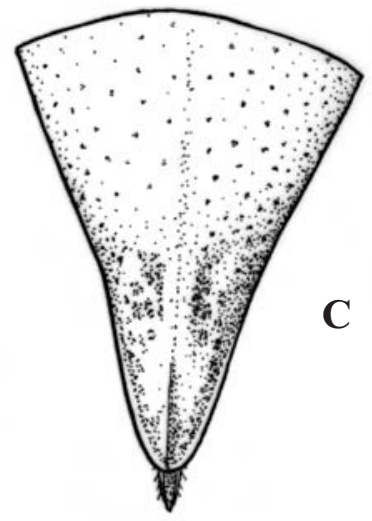

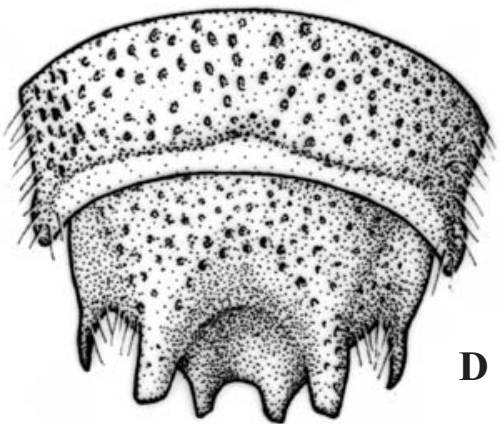

Fig. 9.- Coelioxys (C.) lanceolata: extremo del gáster de la ㅇ (A) y del O (B); C. (C.) mandibularis: ídem (C, D).

Fig. 9.- Coelioxys (C.) lanceolata: apex of the gaster of the 우 (A) and of the (B); C. (C.) mandibularis: the same (C, D).

9. Área supraclipear y frente (entre las inserciones antenales) con una quilla central longitudinal. Mandíbulas rojizas, antenas a lo sumo parduscas. $\mathrm{T}_{1}$ negro. $\mathrm{E}_{6}$ moderadamente estrechado, con una escotadura redondeada en el extremo (Fig. 2D). Longitud: 6,5-9 mm

C. (A.) afra

- Área supraclipear y frente (entre las inserciones antenales) ligeramente abombadas pero sin quilla. Mandíbulas y antenas pardo rojizas. $\mathrm{T}_{1}$ rojo. $\mathrm{E}_{6}$ abruptamente truncado en el extremo apical (Fig. 5C). Longitud: 7-9 mm

C. (A.) haemorrhoa

\section{Subgénero Coelioxys}

\section{Machos}

1. $\mathrm{E}_{4}$ presenta una escotadura en el centro del borde apical. Ángulos distales de $\mathrm{T}_{5}$ normalmente no espinosos ........ 2

- $\mathrm{E}_{4}$ sin escotadura en el centro del borde apical. Ángulos distales de $\mathrm{T}_{5}$ normalmente en espina

2. Terguitos gastrales con amplias manchas triangulares de pelos blancos en posición lateral. $\mathrm{T}_{6}$ provisto de espinas laterales romas; las centro-superiores rectangulares, dos veces más anchas que las centro-inferiores. $T_{5}$ con los ángulos distales formando espina triangular roma cuyo extremo no queda separado del terguito (Fig. 7F). Espolones tibiales externos del tercer par de patas rectos y anchos. Longitud: $12-14 \mathrm{~mm}$

C. (C.) conoidea

- Terguitos con bandas distales (la primera puede estar diluida en el centro). $T_{6}$ provisto de espinas laterales agudas; las centrales superiores redondeadas o triangulares, de igual anchura que las centrales inferiores o más anchas (Figs. $11 \mathrm{~B}$ y $11 \mathrm{D})$. $\mathrm{T}_{5}$ con los ángulos distales no espinosos. Espolones tibiales externos del tercer par de patas curvados, más estrechos

3. $\mathrm{T}_{2}$ a $\mathrm{T}_{4}$ provistos de bandas distales completas, algo estrechadas en el centro; la de $T_{1}$ generalmente interrumpida, quedando sólo una mancha triangular a cada lado. $\mathrm{T}_{5}$ ligeramente saliente hacia afuera en ángulo obtuso a cada lado; las espinas centrales superiores de $\mathrm{T}_{6}$ son anchas, triangulares; las centrales inferiores ligeramente divergentes, de lados paralelos y redondeadas en su extremo (Fig. 11D). $T_{1}$ presenta pelos escasos en el centro y algo más abundantes a los lados. En $\mathrm{E}_{5}$, antes del borde, que también está escotado, existe una marcada depresión longitudinal corta (Fig. 11E). Longitud: 10-12 mm

C. (C.) rufescens 

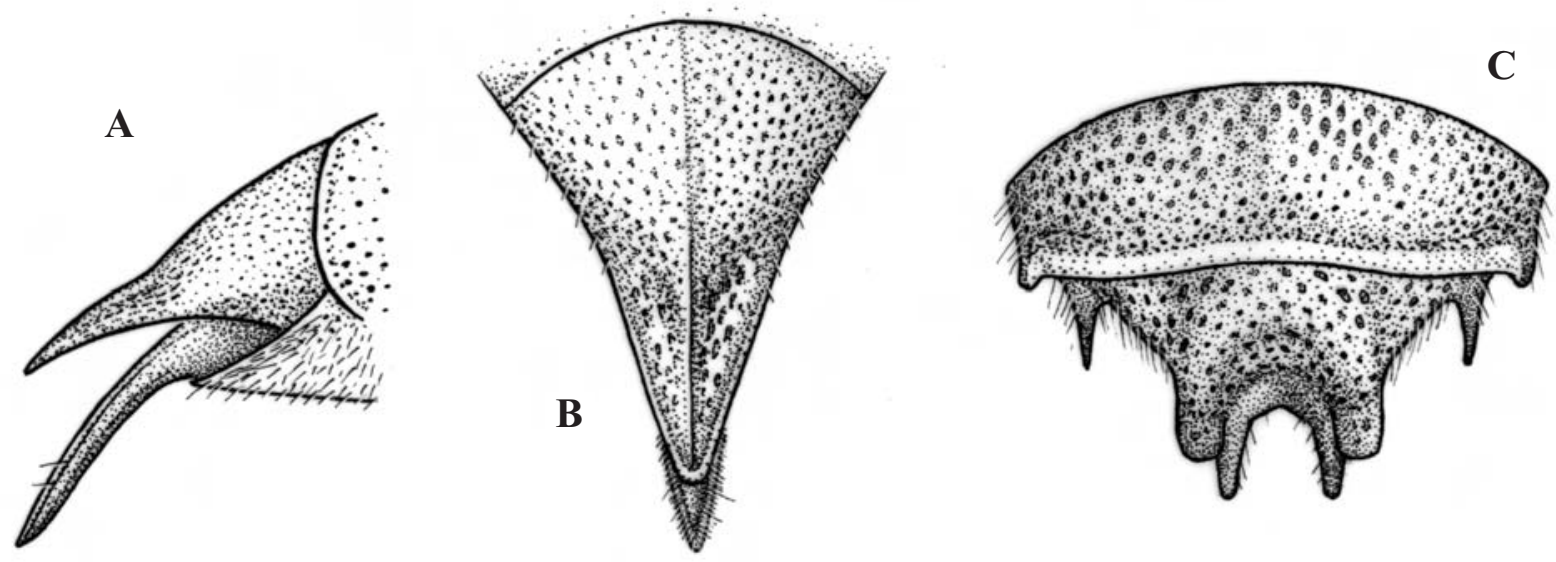

Fig. 10.-Coelioxys (C.) osmiae: extremo del gáster de la 우 (A, B) y del $\sigma^{\pi}(\mathrm{C})$.

Fig. 10.-Coelioxys (C.) osmiae: apex of the gaster of the 9 (A, B) and of the $\sigma^{\pi}(\mathrm{C})$.

- $\mathrm{T}_{2}$ a $\mathrm{T}_{5}$ provistos de bandas distales completas, no estrechadas en el centro; la de $\mathrm{T}_{1}$ generalmente completa, uniendo las manchas triangulares laterales. $\mathrm{T}_{5}$ a los lados no prolongado en ángulo; espinas centrales superiores de $T_{6}$ más estrechas y más largas, las centrales inferiores ligeramente convergentes y redondeadas en su extremo (Fig. 11B). $T_{1}$ con abundantes pelos largos. $E_{5}$ diferente. Longitud: 9-11 mm

C. (C.) quadridentata

4. Tegumento, entre las inserciones antenales, provisto de una línea longitudinal lisa a modo de quilla roma. $\mathrm{T}_{6}$ con una leve quilla longitudinal en el centro de la concavidad, que termina en un diminuto diente triangular (excepcionalmente puede faltar) entre las espinas centro-inferiores; $T_{5}$ con un pequeño diente bilobulado en cada ángulo distal (Fig. 7C). $T_{2}$ con una fóvea estrecha y larga a cada lado y detrás una estrecha zona lisa y brillante. Longitud: $10-12 \mathrm{~mm}$....

C. (C.) aurolimbata algeriensis

- Sin línea longitudinal entre las inserciones antenales. $\mathrm{T}_{6} \sin$ quilla longitudinal en el centro ni diente triangular entre las espinas centro-inferiores; $T_{5}$ provisto o no de un diente grande a cada lado. $\mathrm{T}_{2}$ con una fóvea alargada a cada lado o con una amplia depresión transversal a modo de surco central grande

5. $\mathrm{T}_{2}$ muy densamente punteado y sin fóvea lateral par; provisto de una amplia depresión central transversal que, en el centro, se prolonga ligeramente hacia atrás en sentido longitudinal. Extremo del gáster como en la figura 9B. Longitud: $10-12 \mathrm{~mm}$

C. (C.) lanceolata

- $\mathrm{T}_{2}$ con una fóvea a cada lado. Longitud: 8-12 mm ........ 6

6. Terguitos gastrales con bandas distales completas (pueden quedar diluidas en el centro). $\mathrm{T}_{2}$ provisto de una fóvea corta y oval a cada lado (doble de larga que ancha) (Fig. $8 \mathrm{~F}) . \mathrm{T}_{5}$ con un reborde triangular alargado o con un diente grande romo a cada lado (Fig. 8D). Espolones tibiales del tercer par de patas de color castaño amarillento. En la genitalia, el gonostilo se ensancha en el extremo y está provis- to de pelos que constituyen un tupido pincel (Fig. 8E). Longitud: $11-13 \mathrm{~mm}$................................ C. (C.) elongata

- Terguitos con bandas distales interrumpidas. $T_{2}$ provisto de una fóvea larga y oval a cada lado (unas 3-4 veces más larga que ancha) (Fig. 8K). $\mathrm{T}_{5}$ con un reborde inferior alargado oval o con un diente a cada lado. Espolones tibiales del tercer par de patas de color castaño amarillento o negro. Genitalia sin tupido pincel de pelos (Fig. 8J). Especies algo menores $(8-11 \mathrm{~mm})$

7. Espolones tibiales del tercer par de patas de color castaño amarillento. Basitarso del tercer par de patas 4 veces más largo que ancho. $\mathrm{T}_{6}$ provisto de una pubescencia blanca apretada en la base; espinas centrales superiores casi rectangulares, cortas; las centrales inferiores son largas y estrechas (más de dos veces más largas que las centrales superiores; Fig. 8I). Longitud: 9-10 mm .. C. (C.) inermis

- Espolones tibiales del tercer par de patas de color negro. Basitarso del tercer par de patas 3 veces más largo que ancho. Longitud: 8-10 $\mathrm{mm}$ 8

8. Cara con pilosidad blanco grisácea algo amarillenta. En $\mathrm{T}_{6}$, las espinas centrales superiores son rectangulares largas, más agudas y algo oblícuas al plano horizontal, de igual longitud que las centrales inferiores (Fig. 9D)

\section{C. (C.) mandibularis}

- Cara con pilosidad blanco nívea. En T, las espinas centrales superiores son rectangulares cortas y paralelas al plano horizontal, más cortas que las centrales inferiores (Fig. 10C)

C. (C.) osmiae

\section{Hembras}

1. $\mathrm{E}_{6}$ poco más largo que $\mathrm{T}_{6}$. Ambos son robustos y con el extremo poco agudo. $\mathrm{E}_{6}$ provisto de dos dientes pequeños subapicales (Fig. 11C) o sin ellos (Fig. 7A). Espolones tibiales del tercer par de patas negros 

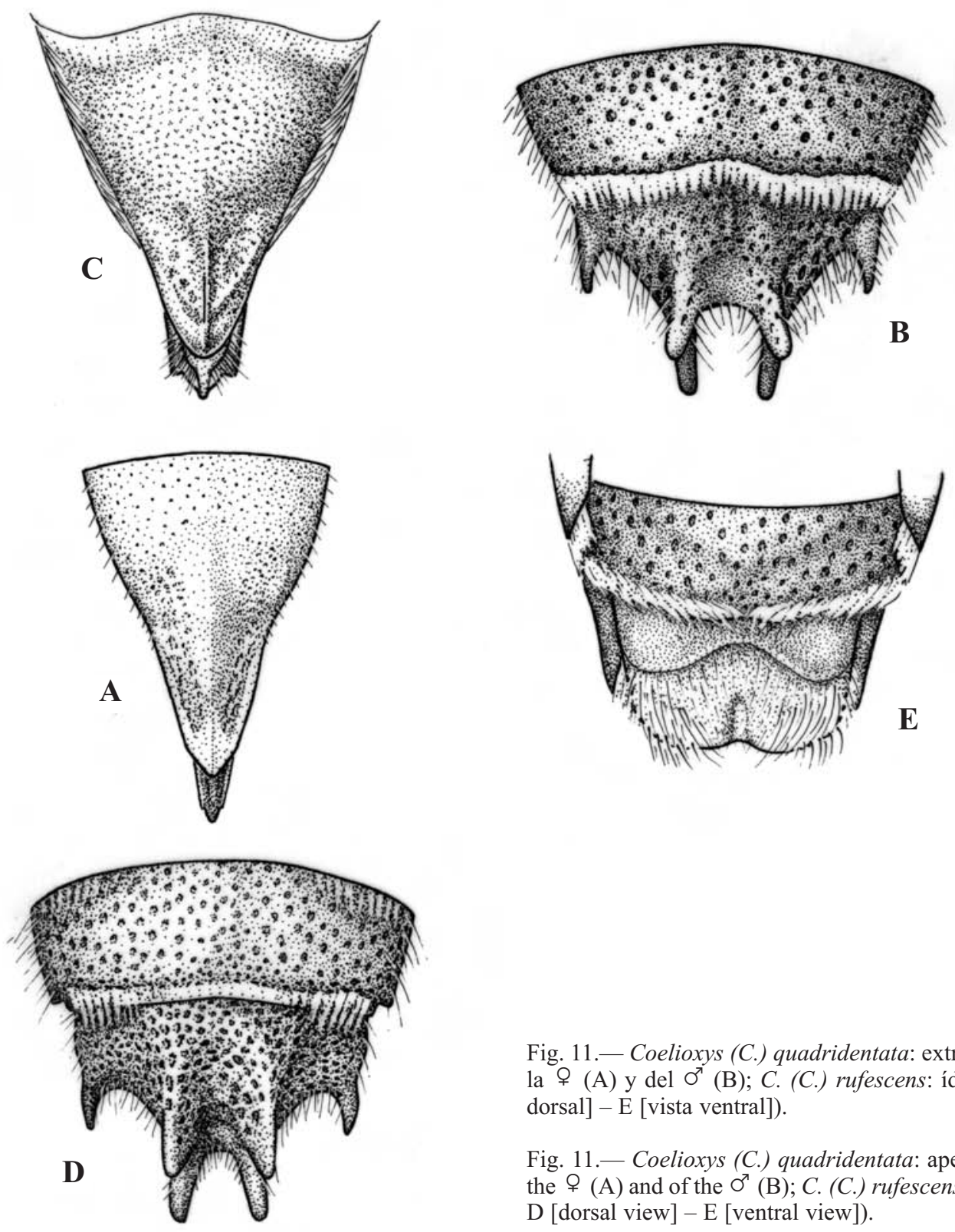

Fig. 11.-Coelioxys (C.) quadridentata: extremo del gáster de la 우 (A) y del O (B); C. (C.) rufescens: ídem (C y D [vista dorsal] - E [vista ventral]).

Fig. 11.- Coelioxys (C.) quadridentata: apex of the gaster of the of (A) and of the $\sigma^{\top}(\mathrm{B}) ; C$. (C.) rufescens: the same (C and $\mathrm{D}$ [dorsal view] - $\mathrm{E}$ [ventral view]).

- Prolongación de $\mathrm{E}_{6}$ claramente 1,5-2 veces más larga que la de $\mathrm{T}_{6}$. En el caso de que sólo sea un poco más larga, sin dientes laterales. Espolones tibiales del tercer par de patas negros o de color pardo amarillento 3

2. $\mathrm{T}_{6}$ con el extremo más agudo, algo ganchudo hacia el dorso del cuerpo y con un reborde acanalado; quilla longitudinal bien marcada y, en el tramo distal, aguda (Figs. 7A y 7B). $\mathrm{E}_{6}$ con el extremo aguzado progresivamente y sin dientes laterales cortos, sólo ángulos obtusos (Fig. 7A). La banda distal de $\mathrm{T}_{1}$ es completa. Clípeo cubierto de tomento corto y denso, apretado, de color blanco algo grisáceo, y fleco distal del mismo color; su extremo apical está amplia y

suavemente arqueado. Longitud: 11-14 mm

\section{C. (C.) aurolimbata algeriensis}

- $\quad \mathrm{T}$ más redondeado en el extremo y con el ápice horizontal y $\sin$ reborde (Fig. 11C); quilla longitudinal sólo superficial. $\mathrm{E}_{6}$ con el ápice triangular corto, aguzado más abruptamente, y con dos dientes laterales en ángulo recto (Fig. 11C). La banda distal de $\mathrm{T}_{1}$ está ampliamente interrumpida. Clípeo con largos pelos laxos de color blanco amarillento algo rojizo; su margen distal con dentículos. Longitud: $10-13,5 \mathrm{~mm}$

C. (C.) rufescens

3. $\mathrm{E}_{6}$ sin contorno continuo, al presentar un par de pequeños dientes laterales (Figs. 8A, 8G, 9C y 11A) 
A

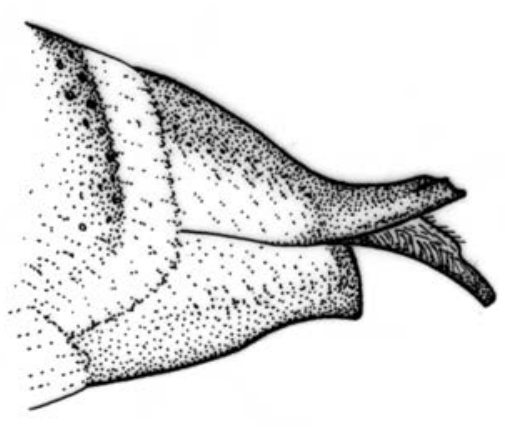

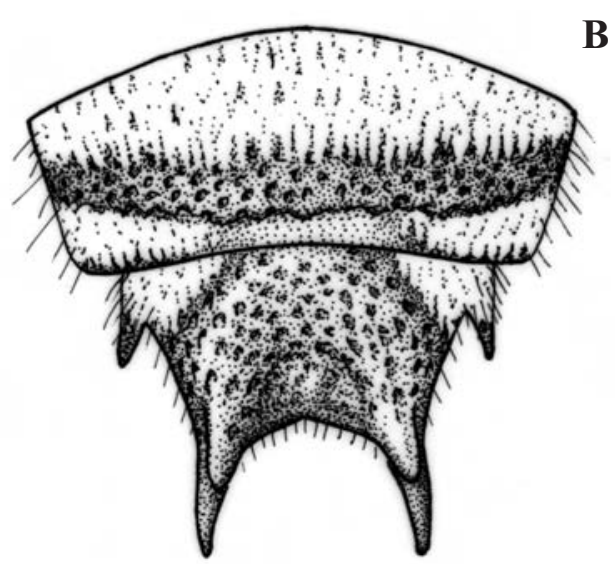

Fig. 12.- Coelioxys (M.) argentea: extremo del gáster de la 우 (A) y del O $\mathrm{O}^{\top}$ (B).

Fig. 12.-Coelioxys (M.) argentea: apex of the gaster of the $ᄋ$ (A) and of the $\sigma^{7}(\mathrm{~B})$.

- $\quad \mathrm{E}_{6}$ con contorno continuo, sin estrechamiento marcado por dientes laterales (Figs. 7D, 9A y 10B) 7

4. Mandíbulas robustas, acodadas en ángulo recto en su borde superior, con pubescencia densa y corta de color castaño. $\mathrm{E}_{5}$ en el extremo truncado o provisto de una escotadura central, con un flequillo distal denso de color castaño amarillento; su margen distal tan ancho como la base de la prolongación de $\mathrm{E}_{6}$; extremo del gáster como en la figura 9C. Longitud: $10-12 \mathrm{~mm}$

C. (C.) mandibularis

- Mandíbulas normales o algo angulosas, pero sin formar un ángulo recto, con pubescencia pálida. $\mathrm{E}_{5}$ en el extremo redondeado, truncado o ligeramente escotado; su margen distal más estrecho que la base de la prolongación de $\mathrm{E}_{6} .$.

5. $\mathrm{T}_{2} \mathrm{y} \mathrm{T}_{3}$ provistos de una zona transversal en gran parte desprovista de puntos (Fig. 8C). Diente central de $\mathrm{E}_{6}$ alargado $\mathrm{y}$ estrechamente redondeado en el extremo, unas 2 veces más largo que su anchura basal; los dientes laterales son pequeños pero espinosos; $\mathrm{T}_{6}$ anchamente redondeado en el extremo (Fig. 8A). $\mathrm{T}_{2}$ a $_{5}$ con una banda distal de pelos. En el tórax, las líneas parapsidales y medial del escudo están desarrolladas en quilla sobresaliente, lo que es más patente en el extremo de la medial. Quilla del diente medio de las mandíbulas fuertemente desarrollada (Fig. 8B). Longitud: $12-14 \mathrm{~mm}$ C. (C.) elongata

- Zona transversal lisa de $\mathrm{T}_{2} \mathrm{y} \mathrm{T}_{3}$ interrumpida, sobre todo en el centro, con puntos más o menos dispersos y gruesos. Las líneas parapsidales y medial no forman ninguna quilla. En las mandíbulas, quilla del diente medio no fuertemente diferenciada. Extremo del gáster como en las figuras $8 \mathrm{G}$ y 11A. Especies menores

6. $\mathrm{T}_{2}$ en el centro del disco con punteado medio denso y profundo (Fig. $8 \mathrm{H}$ ). $\mathrm{E}_{6}$ uniformemente estrechado hasta su ápice, de estrechamente redondeado a agudo; dientes cen- tral y laterales en ángulo recto y más o menos romos (Fig. $8 \mathrm{G})$. Borde del clípeo con un fleco de pelos rojo-amarillentos. $\mathrm{T}_{2}$ a $\mathrm{T}_{4}$ con una banda distal ancha interrumpida en el centro; $T_{5} \sin$ banda apical. Espolones tibiales del tercer par de patas de color castaño amarillento. Longitud: 9-12 $\mathrm{mm}$

\section{C. (C.) inermis}

- $\mathrm{T}_{2}$ desprovisto de puntos en gran parte del centro del disco. $\mathrm{E}_{6}$ fuertemente estrechado en la mitad de su longitud apical, con el ápice estrechamente redondeado de lados paralelos; $\mathrm{E}_{6}$ casi de igual anchura que la parte distal de $\mathrm{T}_{6}$ (Fig. 11A). $\mathrm{E}_{5}$ estrechamente redondeado en su extremo. Borde del clípeo con un fleco de pelos de color blanco grisáceo. Espolones tibiales del tercer par de patas negros. Longitud: 9,5-12 $\mathrm{mm}$

C. (C.) quadridentata

7. $\mathrm{T}_{2}$ a $\mathrm{T}_{4}$ provistos de banda distal entera. $\mathrm{T}_{6}$ con el ápice semicircular; E6 con forma de hoja de lanza (Fig. 9A). Espolones tibiales externos del tercer par de patas de color pardo amarillento, agudos en el extremo. Longitud: 11-13 $\mathrm{mm}$

C. (C.) lanceolata

- $\mathrm{T}_{1}$ a $\mathrm{T}_{5}$ provistos de una mancha triangular blanca a cada lado. $\mathrm{T}_{6}$ con el ápice más estrecho (Fig. 10B) o ligeramente truncado (Fig. 7D). Espolones tibiales del tercer par de patas negros

\section{8}

8. Espolones tibiales externos del tercer par de patas rectos y anchos, romos en su extremo (Fig. 7E). $\mathrm{T}_{6}$ ancho, truncado en el extremo; $\mathrm{E}_{6}$ ancho y fuertemente apuntado antes del extremo (Fig. 7D). Longitud: 13-15 mm

C. (C.) conoidea

- Espolones tibiales externos del tercer par de patas con los lados convergentes y estrechos, agudos en su extremo. $\mathrm{T}_{6} \mathrm{y}$ $\mathrm{E}_{6}$ más estrechos, progresivamente estrechados hasta el extremo (Fig. 10B). Longitud: 9,5-11 mm

C. (C.) osmiae 


\section{Subgénero Mesocoelioxys}

Sólo una especie: $\boldsymbol{C}$. (M.) argentea (Fig. 12).

\section{AGRADECIMIENTOS}

A Maximilian Schwarz (Ansfelden, Austria), por el amable préstamo de ejemplares de su colección particular y distintos comentarios sobre el género Coelioxys. Los dibujos han sido realizados por Iñaki Díez Cortaberría (Fig. 1) y Alexandre Anichtchenko (Figs. 2-12). Las figuras 2F, 3C, 8C, 8F, 8H y $8 \mathrm{~K}$ han sido cedidas por Erwin Scheuchl (Ergolding, Alemania).

\section{Referencias}

Amiet, F., Herrmann, M., Müller, A. \& Neumeyer, R., 2004. Fauna Helvetica 9. Apidae 4. Centre suisse de cartographie de la faune. Neuchâtel. 273 pp.

BANASZAK, J. \& Romasenko, L., 2001. Megachilid bees of Europe (Hymenoptera, Apoidea, Megachilidae). Pedagogical University of Bydgoszcz. Bydgoszcz. $239 \mathrm{pp}$.

Dusmet, J. M., 1906. Los Ápidos de España. II. Género Coelioxys. Boletín de la Real Sociedad española de Historia Natural, 6: 134-151.

Michener, C. D., 2007. The bees of the World. Second edition. The Johns Hopkins University Press. Baltimore. xvi $+953 \mathrm{pp}$.

Ornosa, C., Ortiz-Sánchez, F. J. \& Torres, F., 2007. Catálogo de los Megachilidae del Mediterráneo occidental (Hymenoptera, Apoidea). II. Lithurgini y Megachilini. Graellsia, 63(1): 113-134.

PřIdAL, A. \& TKALCŮ, B., 2001. Commentary to the subgenera of the genus Coelioxys described by Ruszkowski 1986 (Hymenoptera: Apoidea: Megachilidae). Entomofauna, 22(14): 357-364.
SCHEUCHL, E., 1996. Illustrierte Bestimmungstabellen der Wildbienen Deutschlands und Österreichs. Band II: Megachilidae - Melittidae. Eigenverlag. Velden. $116 \mathrm{pp}$.

SCHWARZ, M., 1999. Bemerkungen, Korrekturen und Ergänzungen zu den von Gupta (1993) 1999 in "Taxonomic Studies on the Megachilidae of NorthWestern India" behandelten Coelioxys-Arten (Hymenoptera, Apidae). Entomofauna, 20(8): 145162.

Schwarz, M., 2009. Eine für Europa neue CoelioxysArt: Coelioxys osmiae Alfken 1928 (Hymenoptera: Apidae: Megachilidae). Entomofauna, 30(29): 493501.
Recibido, 5-X-2009

Aceptado, 23-XI-2009 Publicado, 31-XII-2009 